\section{AMPLIANDO NUESTRA COMPRENSIÓN SOBRE LA CREACIÓN Y REDUCCIÓN DEL RIESGO DE DESASTRES EN LATINOAMÉRICA Y EL CARIBE}

Ricardo Mena ${ }^{1, *}$

\section{RESUMEN}

Este prólogo presenta el 3er número (Vol. 2, Núm. 2) de la Revista de Estudios Latinoamericanos sobre Reducción del Riesgo de Desastres (REDER) e introduce sus articulos, que son el resultado de investigaciones recientes, así como reflexiones metodológicas sobre las variadas dimensiones sociales, económicas, políticas y ambientales que interactúan con los procesos de creación y reducción del riesgo de desastre en la región. Las investigaciones se relacionan de manera directa con las áreas de acción propuestas por el Marco de Sendai, el plan global para la reducción del riesgo de desastres adoptado en el año 2015 por los estados miembros de las Naciones Unidas, que tiene como propósito prevenir la aparición de nuevos riesgos, disminuir los existentes e implementar medidas para reforzar la resiliencia.

\section{PALABRAS CLAVE}

Reducción del riesgo de desastres, Gestión del riesgo, Resiliencia, Marco de Sendai

\section{UNDERSTANDING THE CREATION AND REDUCTION OF DISASTER RISKS IN LATIN AMERICA AND THE CARIBBEAN}

\section{ABSTRACT}

This preface presents the 3rd Issue (Vol. 2, No. 2) of the Journal of Latin American Studies on Disaster Risk Reduction (Revista de Estudios Latinoamericanos sobre Reducción del Riesgo de Desastres REDER) and introduces its articles. The research articles are the result of recent investigations as well as methodological reflections on the various social, economic, political and environmental dimensions embedded within the processes of creation and reduction of disaster risks in the region. All articles are directly related to the areas of action proposed by the Sendai Framework, the global plan for disaster risk reduction adopted in 2015 by the United Nations member states, whose purpose is to prevent the occurrence of new risks and reduce existing ones and implement measures to strengthen resilience.

\section{KEYWORDS}

Disaster risk reduction, Risk management, Resilience, Sendai Framework
1. Jefe de la Subdivisión de Apoyo y Monitoreo para la Implementación del Marco de Sendai de la Oficina de las Naciones Unidas para la Reducción del Riesgo de Desastres (UNISDR), Ginebra, Suiza.

*Autor de correspondencia: menar@un.org

Formato cita Recomendada (APA):

Mena, R. (2018). Ampliando nuestra comprensión sobre la creación y reducción del riesgo de desastres en Latinoamérica y el Caribe. Revista de Estudios Latinoamericanos sobre Reducción del Riesgo de Desastres REDER, 2(2), pp.1-3

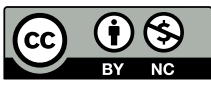

Todos los artículos publicados en REDER siguen una política de Acceso Abierto y se respaldan en una Licencia CreativeCommons Atribución-NoComercial 4.0 Internacional.

Revista de Estudios Latinoamericanos sobre Reducción del Riesgo de Desastres (REDER)

Diseño: Lupe Bezzina Tipografía: Hospital 


\section{PRÓLOGO}

Este nuevo número de la Revista de Estudios Latinoamericanos sobre Reducción del Riesgo de Desastres (REDER) presenta resultados inéditos de investigaciones recientes, así como reflexiones metodológicas sobre las variadas dimensiones sociales, económicas, políticas y ambientales que interactúan con los procesos de creación y reducción del riesgo de desastre en la región. Tal y cómo ha sido la política de esta revista desde sus comienzos, las investigaciones de este número presentan distintos enfoques disciplinares e interdisciplinares, situadas desde marcos metodológicos diversos. La naturaleza de las reflexiones que nos presentan estas investigaciones nos invita a ampliar nuestra comprensión de la problemática de los riesgos en la región, desde la efectividad de ciertas viviendas de emergencia en Chile, hasta estudios de desempeño del sector público del caribe mexicano respecto a la gestión del riesgo de desastres.

Las investigaciones se relacionan de manera directa con las cuatro áreas de acción propuestas por el Marco de Sendai (UNISDR, 2015), el plan global para la reducción del riesgo de desastres adoptado en el año 2015 por los estados miembros de las Naciones Unidas, que tiene como propósito prevenir la aparición de nuevos riesgos, disminuir los existentes e implementar medidas para reforzar la resiliencia. Sus cuatro áreas de acción abordan aspectos relacionados con (1) la necesidad de comprender mejor el riesgo de desastres en todas sus dimensiones; (2) el fortalecimiento de la gobernanza para gestionar el riesgo de desastres; (3) el incremento de las inversiones en reducción del riesgo de desastres para fortalecer la resiliencia de las personas; $y_{1}(4)$ aumentar la preparación para casos de desastres a fin de dar una respuesta eficaz y "reconstruir mejor" en los ámbitos de la recuperación, rehabilitación y la reconstrucción.

La necesidad de comprender mejor el riesgo de desastres es abordada por los autores Marin, Méndez y Sarmiento, quienes nos hacen una interesante invitación a releer la precariedad urbana en Latinoamérica y el Caribe desde una perspectiva de reducción del riesgo. A través de métodos cualitativos, los autores realizan una revisión exhaustiva de 23 modelos de medición de precariedad urbana, con el objetivo de caracterizar su multicausalidad, multidimensionalidad e identificar las tendencias de uso de las mismas. Entre sus principales observaciones está que las dimensiones físicas, socioeconómicas, sociopolíticas, y legales de la precariedad urbana no están siempre relacionadas, de forma directa, a factores de riesgo. Sin embargo, las relaciones entre estas dimensiones pueden favorecer y potenciar la generación de riesgos, lo cual a su vez puede acentuar condiciones de precariedad, concluyendo con esto los autores, que precariedad y riesgo urbano no deberían ser analizados aisladamente.

Por su parte, Jon Cadierno Gutiérrez explora la transmisión de conocimiento ancestral dentro de la comunidad indígena mapuche-lavkenche, en Chile, como una práctica cultural relevante para la resiliencia social, en especial respecto a la capacidad de respuesta rápida ante terremotos y maremotos. Cadierno Gutiérrez utiliza entrevistas semi-estructuradas, y la posterior aplicación de la Teoría Fundamentada con ayuda de la herramienta Atlas.ti, para constatar que la transmisión de dicho conocimiento ocurre a través de historias y de experiencias directas que toman lugar en el seno de las familias, las comunidades y los colegios, suponiendo una vía efectiva de comunicación del riesgo y la más relevante fuente de educación sobre el riesgo. El autor concluye que las advertencias de los mayores, sabios y líderes comunitarios, la propia cultura, el arraigo territorial, el estrecho vínculo con la naturaleza y la fe en las historias y otras creencias se unen para facilitar una percepción certera del riesgo y sus consecuentes comportamientos preventivos.

Posteriormente, Garay, Tapia, Castillo, Fernández y Vergara nos presentan reflexiones de una investigación aplicada relacionada al uso de la madera para viviendas de emergencia y asentamientos transitorios luego de desastres en el contexto chileno. Este trabajo indaga y trae a la discusión normativas de la región y las compara con la normativa de Chile, la cual, según los autores, no aborda suficientemente las características de combustibilidad de los materiales de construcción. A partir del análisis, los autores plantean la importancia de una reestructuración de las normativas existentes, de manera que se adecúe a las exigencias de los distintos criterios técnicos y 
que contemple la necesidad de normalizar los tipos de materiales estructurales y de revestimiento que se deben evitar en estructuras destinadas a ciertos usos con el objeto de minimizar el riesgo de incendio, su velocidad de propagación, toxicidad, entre otros factores. Los autores finalizan con una propuesta de índice integrado que sería capaz de medir diferentes niveles de seguridad y sostenibilidad en el uso de la madera como material adecuado para su uso durante las reconstrucciones pos-desastres.

Por otro lado, la investigación de Rosalía Chávez Alvarado busca explicar mejor el papel que tienen las instituciones de gobierno municipal en ciudades costeras del caribe mexicano respecto a la gestión del riesgo de desastre. A través de entrevistas y observaciones en terreno, los resultados de esta investigación permitieron a Chávez Alvarado reflexionar sobre las trayectorias existentes de desarrollo urbano en Quintana Roo, México. La autora concluye que, a pesar de la legislación existente, la cual pretende apoyar procesos de desarrollo urbano sostenibles y resilientes, la resiliencia de las comunidades y del entorno urbano parece desarrollarse muy lentamente y con limitaciones, siendo la 'permisividad' uno de los factores que favorecen acciones peligrosas. Sin responsables jurídicos, razona Chávez Alvarado, se sostiene un proceso de adaptación de la población a perder fauna, flora, ecosistemas y al aumento de su fragilidad al entorno.

Finalmente, la autora Daniela Aragón Urtubia nos invita a revisar los resultados de su investigación sobre la construcción social del riesgo urbano y rural en Putre, Chile. Desde el marco conceptual propuesto por el modelo de Presión y Liberación de Blaikie et al. (1994), Aragón Urtubia utiliza la herramienta de Análisis de Vulnerabilidades y Capacidades (AVC), en conjunto con entrevistas en profundidad sobre la percepción individual, para adentrarse en los diferentes factores sociales que interactúan para construir el riesgo en la localidad estudiada. Basada en los resultados, la autora establece diferencias en la construcción del riesgo entre entornos urbanos y rurales, en donde aspectos como la participación, institucionalidad y gobernanza alcanzan una especial relevancia.

\section{REFERENCIAS}

United Nations International Strategy for Disaster Reduction UNISDR. (2015). Sendai Framework for Disaster Risk Reduction 2015-2030. Sendai: UNISDR.

Blaikie, P., Terry Cannon, Ian Davis, \& Ben Wisner. (1994). At risk: natural hazards, people's vulnerability, and disasters. London: Routledge. 